\title{
Super-Humeanism: the Canberra plan for physics
}

\author{
Michael Esfeld \\ University of Lausanne, Department of Philosophy \\ Michael-Andreas.Esfeld@unil.ch,www.michaelesfeld.com \\ (draft 5 November 2017)
}

(for Anna Marmodoro (ed.): The foundation of reality: fundamentality, space and time.

Oxford: Oxford University Press)

\begin{abstract}
The paper argues for a metaphysics in the vein of the Canberra plan, namely to single out a minimal, basic set of entities and then to show how everything else is located in that set by being identical with something in that set and how the propositions that describe the basic entities entail all the other true propositions. The paper conceives the Canberra plan for the domain of the natural sciences as a naturalized metaphysics that is not committed to a priori entailment. The proposal is that the minimal set of entities is defined by the following two axioms: (1) There are distance relations that individuate simple objects, namely matter points. (2) The matter points are permanent, with the distances between them changing. Finally, the paper explains how the Canberra plan sets a clear standard for ontological issues that go beyond the natural sciences.
\end{abstract}

Keywords: Canberra plan, minimalist ontology, parsimony, Humeanism, Super-Humeanism, relationalism about space-time, ontic structural realism

\section{The Canberra plan}

Consider how Jackson (1994, p. 25) describes the task of metaphysics:

Metaphysics, we said, is about what there is and what it is like. But of course it is concerned not with any old shopping list of what there is and what it is like. Metaphysicians seek a comprehensive account of some subject matter - the mind, the semantic, or, most ambitiously, everything - in terms of a limited number of more or less basic notions. In doing this they are following the good example of physicists. The methodology is not that of letting a thousand flowers bloom but rather that of making do with as meagre a diet as possible. ... But if metaphysics seeks comprehension in terms of limited ingredients, it is continually going to be faced with the problem of location. Because the ingredients are limited, some putative features of the world are not going to appear explicitly in the story. The question then will be whether they, nevertheless, figure implicitly in the story. Serious metaphysics is simultaneously discriminatory and putatively complete, and the combination of these two facts means that there is bound to be a whole range of putative features of our world up for either elimination or location.

This quotation can be considered as a locus classicus of what is known as the Canberra plan: metaphysics is ontology, answering the question of what there is in such a way that something is admitted as fundamental and it is then shown how everything else that exists is included in what is fundamental (location). This implies that the propositions that describe what is fundamental entail all the other true propositions about the world. 
This paper sets out a proposal how to implememt a Canberra plan for the physical domain. In doing so, the proposal made here deviates in three main aspects from positions that are connected with the Canberra plan. (1) In the first place, as far as the methodology is concerned, this paper is an essay in metaphysics of science or naturalized metaphysics rather than a priori metaphysics ("armchair metaphysics"). When it comes to a concrete proposal about what is fundamental in the world, the benchmark is not only to take science, notably physics, into consideration, but to show in precise terms how the proposal in question matches our central physical theories. This benchmark amounts to a naturalization of metaphysics. However, it does not mean returning to positivist metaphysics. There is no question of reading the proposal for what is fundamental off from the mathematical structure of physical theories (e.g. by translating this structure into first order logic and taking the propositions with existential quantifiers thus obtained to settle the ontological commitments, as in Quine's vision of metaphysics, see Quine 1948). Formulating such a proposal is genuine philosophical work, the guideline being "making do with as meagre a diet as possible", as Jackson puts it. That is, the criterion is parsimony, together with coherence and empirical adequacy. In other words, the question that naturalized metaphysics, thus conceived, seeks to answer is this one: What is a minimal set of entities that form an ontology of the natural world, given what science tells us about the natural world? In a nutshell, the criterion is minimal sufficency, neither necessity, nor a priori knowledge.

(2) Moreover, taking recent developments in metaphysics into account that have moved away from the notion of supervenience, "location" is in the following conceived in terms of identity. The task hence is to spell out what there is in terms of a minimal set of entities and then to show how everything else is located in that set in being identical with something in that set. The advantage of working with the notion of identity is that it is clear and simple: if $A$ and $B$ exist, then $B$ is either identical with $A$ or not identical with $A$. If $B$ is identical with $A$, then endorsing $B$ requires no ontological commitment that goes beyond $A$-given $A, B$ comes for free. If $B$ exists and is not identical with $A$, then $B$ is something in addition to $A$. Hence, an ontological commitment going beyond $A$ is necessary. Thus, if $A$ is the set of entities originally admitted as minimal, that set then has to be enlarged to include $B$. Working with identity instead of supervenience is no deviation from the Canberra plan as set out in Jackson or in Lewis's metaphysics of Humean supervenience, since for them, in brief, supervenience is a vehicle to establish identity and ontological reduction of everything else to the supervenience basis (e.g. Lewis 1986a, introduction, and Jackson 1998, ch. 1).

The fact that identity is symmetrical does not infringe upon the ontological reductionism of locating everything in a minimal set of entities. Suppose that the configuration of point particles of the universe is that minimal set of entities - that is, all the point particles of the universe conceived as permanent with their relative positions (i.e. the distances among these particles) and their motion (i.e. the change of their relative positions, that is, the change of their distances). The basic notions then are "point particle", "distance" and "motion" (i.e. "change of distance"). The problem of location then is to show how everything else is identical with some specific sub-configuration of the universal configuration of particles and its motion. Suppose that organisms, including minds, exist. The task then is to spell out by using only the basic notions of point particles, distances and change of distances which specific configurations of particles and their motions are organisms and which ones are minds. If this cannot be done, more than particles standing in distance relations and the 
change of these relations has to be admitted in the ontology. Hence, the reductionism consists in establishing that the configuration of particles of the universe is everything that exists, and this is done through the symmetry of the identity relation by showing that some subconfigurations of particles of the universe are organisms so that organisms are certain specific particle configurations, some further sub-configurations are minds so that minds are certain specific particle configurations, etc.

(3) In the third place, ontological reductionism thus spelled out in terms of identity commits one to the view that the propositions describing the minimal set of entities entail all the true propositions about the world. However, there is no commitment to a priori entailment endorsed in this paper. The core idea of the Canberra plan is that given the basic notions that describe the minimal set of entities, all further notions are introduced through a functional definition that describes their causal or functional role for the behaviour of the entities in that set (see e.g. Lewis 1970 and 1972). But it is not mandatory to consider these functional definitions as being a priori, that is, as being the business of conceptual analysis only; they may rather be a matter of scientific investigation. In any case, given these functional definitions, the propositions describing the minimal set of entities then entail all the other true propositions about the world. Thus, supposing that the minimal set of entities is the particle configuration of the universe, if the notions describing the mind can be defined in terms of their causal role for particle motion, then the description of the particle configuration of the universe and its change entails all the true propositions describing minds.

\section{Physics: ontology}

Naturalized metaphysics turns to physics when it comes to determining the minimal set of entities on the basis of which everything in the domain of the natural sciences can be understood. Consider what Feynman says in the introduction to the Feynman lectures on physics:

If, in some cataclysm, all of scientific knowledge were to be destroyed, and only one sentence passed on to the next generations of creatures, what statement would contain the most information in the fewest words? I believe it is the atomic hypothesis (or the atomic fact, or whatever you wish to call it) that all things are made of atoms - little particles that move around in perpetual motion, attracting each other when they are a little distance apart, but repelling upon being squeezed into one another. In that one sentence, you will see, there is an enormous amount of information about the world, if just a little imagination and thinking are applied.

(Feynman et al. 1963, vol. 1, ch. 1-2)

This the old and venerable hypothesis of atomism, going back to the Presocratics Leucippos and Democritos and being turned into a precise physical theory by Newton. The idea is that matter is composed of point particles and that everything in the physical domain is identical with configurations of these point particles. Consequently, all the variation and change in the physical domain can be accounted for in terms of differences in the spatial arrangement of these point particles and the change of their spatial arrangement.

The philosophical task then is to elaborate on the atomic hypothesis in such a way that it is clearly spelled out what the commitment to a minimal set of entities is, what the basic notions are that express that commitment and how all the further notions come in as being defined in terms of their function for that minimal set of entities. In that respect, as is clear since Leibniz' criticism of Newton in the famous Leibniz-Clarke correspondence, the ontological 
commitments to an absolute space in which the atoms are embedded and an absolute time in which their motion takes place amount to endorsing a surplus structure, which shows up, in the case of Newtonian physics, in the guise of a commitment to ontological differences that do not make a physical difference (see notably Leibniz' third letter, §§ 5-6, and fourth letter, $\S$ 15, in Leibniz 1890, pp. 363-364, 373-374, English translation Leibniz 2000). Consequently, on a minimalist ontology, the commitment should only be to spatial relations (i.e. distances) among the point particles as the relations that define, in Leibnizian terms, the order of coexistence of the point particles, and the change of these relations, with time being the measure of that change (order of succession, in Leibnizian terms; see notably third letter, $\S 4$, and fourth letter, $\S 41$, in Leibniz 1890, pp. 363, 376).

By the same token, it amounts to a commitment to a surplus structure to conceive the point particles as being equipped with some intrinsic properties - in other words, to conceive physical parameters such as mass and charge as referring to intrinsic properties of the particles. As, for instance, Mach (1919, p. 241) stresses when commenting on Newton's Principia, "The true definition of mass can be deduced only from the dynamical relations of bodies". That is to say, both inertial and gravitational mass are introduced in Newtonian mechanics through their dynamical role, namely as dynamical parameters that couple the motions of the particles to one another. In general, even if attributed to the particles taken individually, mass, charge, etc. express a dynamical relation between the particles instead of indicating intrinsic properties of the basic objects. Hence, mass, charge, etc. are parameters that can be defined in terms of what they do for the basis objects, namely in terms of their causal or functional role for the motion of the basic objects.

The commitment to surplus structure lies in the fact that if one conceives mass, charge, etc. as intrinsic properties of the particles that are dispositions with the acceleration of the particles being their manifestation, there are situations (including entire possible worlds) conceivable in which these dispositions do not manifest themselves, or situations in which they cancel each other out - such as a situation in which the attractive motion resulting from the gravitational mass of two particles is cancelled out by the repulsive motion resulting from these two particles having the same charge so that there is no relative motion of the two particles. If one conceives mass, charge, etc. as intrinsic, categorical properties of the particles so that the functional role that these properties exercize for the motion of the particles is contingent, the surplus structure then is that one is committed to quidditism, namely to a purely qualitative essence (quiddity) of these properties that is not accessible to physics (humility) (see e.g. Lewis 2009).

Leaving absolute space and time as well as intrinsic properties aside for these reasons, we can formulate atomism as ontology of the natural world in terms of the following two axioms:

(1) There are distance relations that individuate simple objects, namely matter points.

(2) The matter points are permanent, with the distances between them changing.

Consequently, the specific notions endorsed as primitive are the ones of matter points, distances and change of the distances (see Esfeld and Deckert 2017, ch. 2.1, for details).

The reason for singling out the distance relation is that it is the first and foremost candidate for the world-making relation, at least insofar as the physical world is concerned. All and only those objects that stand in a distance to each other make up a world. No other relation has been proposed in the literature that could do the same for the physical world (Lewis's hypothetical basic relations of like-chargedness and opposite-chargedness, for instance, would 
not do, since, as Lewis (1986b, p. 77) notes himself, these relations fail to distinguish the objects that stand in them as soon as there are at least three objects). The world-making character of the distance relation, at least with respect to the physical world, is the reason why Leibniz considers space as the order of coexistence. The distance relation defines extension, which means that the objects that stand in this relation are not extended themselves. They thus are point objects, more precisely matter points, taking up the Cartesian definition of matter as res extensa. In other words, the distance relation is such that it makes it that the objects that stand in this relation are matter points.

The distance relation is irreflexive, symmetric and connex (meaning that all the objects in a given configuration are connected with one another by a relation of this type). It satisfies the triangle inequality (that is, for any three matter points $i, j, k$, the sum of the distances between $i$ and $j$ and $j$ and $k$ is greather than or equal to the distance between $i$ and $k$ ). This is the central metrical requirement. The triangle inequality only implies that the ratios between the distances are part of the ontology, but it does not single out a specific geometry, such as Euclidean, or Riemannian geometry, that the distances have to satisfy. This is an issue of representation, with the change that actually occurs in the distance relations determining which geometry is best suited to represent the distance relations and their change.

Unless one takes the radical ontological structural realism proposed by French and Ladyman to be intelligible (see e.g. Ladyman and Ross 2007, chs. 2 and 3, and French 2014, chs. 5-7) - or, to put it more mildly, maintains that the formulation of ontology calls for a change in first order logic -, relations require objects as that what is related by the relations in question. Consequently, both the relations and the objects that stand in the relations in question are ontologically basic. It is not clear to say the least how relations could constitute objects, or how objects could emerge from relations, in the sense that objects are somehow derived from relations. Objects may be nothing more than the nodes in a network of relations - in the sense that all there is to the objects is that they are points standing in distance relations, they do not have any intrinsic properties, or a haecceity -, but, still, the objects are ontologically distinct from the relations: the points are ontologically distinct from the distances that obtain between them. By the same token, it is, pace Heil (2012, ch. 7) and Lowe (2016), unclear to say the least how there could be only objects making up a world, but no world-making relations that bind these objects together so that they make up a world. No one has put forward a proposal how to derive relations from objects.

Hence, both relations and objects are basic; but one gets the individuation of objects for free from the relations. In other words, given that one has to recognize relations anyway, one can employ the relations endorsed as basic as being that what individuates the objects. One thereby obtains a structural individuation of objects, by contrast to an individuation through an intrinsic essence, a bare substratum, or a primitive thisness (haecceity). Thus, the distance relation can individuate the matter points provided that it fulfills the following requirement: if matter point $i$ is not identical with matter point $j$, then the two sets that list all the distance relations in which these points stand with respect to all the other points in a configuration must differ in at least one such relation. It is such differences in the way in which $i$ and $j$ relate with the other points in the configuration that make it that $i$ and $j$ are different points. Consequently, by imposing this requirement, one avoids having to accept the plurality of matter points as a primitive fact, which would imply that the matter points are bare particulars or bare substrata. The distance relation also accounts for the impenetrability of matter without 
having to invoke a notion of mass: for any two matter points to overlap it would have to be the case that there is no distance between them. This requirement implies that any model or possible world of this ontology has to include at least three matter points that are individuated by the distance relations among them. Consequently, symmetrical configurations are ruled out, but also, for instance, the configuration of an isosceles triangle. Nonetheless, this is no objectionable restriction: having empirical adequacy in mind, there is no need to admit worlds with only one or two objects or entirely symmetrical worlds (see Hacking 1975 and Belot 2001 for also ruling such worlds out as being metaphysically possible).

\section{Physics: representation}

If the ontology is exhausted by matter points standing in distance relations and the change of these relations so that "matter points", "distances" and "change of distances" are the primitive notions, these notions are not sufficient to formulate a physical law. The reason is that a configuration of matter points described in terms of these notions does not contain any information about its evolution. Of course, these notions are sufficient to describe all the change that occurs in the configuration of matter of the universe. But that would just be a description that lists all the change that actually happens by contrast to a law that simplifies that is, a law that is such that given some initial configuration of matter, the law tells us something about the evolution of the configuration (in the case of a deterministic law, it contains the information about the whole past and future evolution of the configuration). In order to achieve such a law, we have to embed the configuration of matter in a geometry and a dynamics: we have to conceive the configuration of matter as embedded in a geometrical space (such as Euclidean space) and we have to attribute parameters to the configuration of matter that are introduced in terms of their functional role for the change in the distance relations among the matter points. These can be parameters that are attributed to the matter points individually (such as mass, momentum, charge), to their configuration (such as total energy, or an entangled wave function), or constants of nature (such as the gravitational constant). They can always remain the same (such as mass and charge) or vary as the distance relations among the matter points change (such as momentum, fields, a wave function). Let us call these parameters and the geometry the "dynamical structure" of a physical theory, and let us use the term "primitive ontology" for the ontology that is defined in terms of the basic, primitive notions on the basis of which the dynamical structure then is introduced in terms of its functional role for that ontology.

Consider how Hall $(2009, \S 5.2)$ describes the task of a physical theory:

... the primary aim of physics - its first order business, as it were - is to account for motions, or more generally for change of spatial configurations of things over time. Put another way, there is one Fundamental Why-Question for physics: Why are things located where they are, when they are? In trying to answer this question, physics can of course introduce new physical magnitudes

- and when it does, new why-questions will come with them.

That is to say: the new parameters - that is, the parameters that go beyond the primitive parameters of relative positions of the basic objects and the change of these positions - come in through the functional role they play for the motion of the basic objects. The benchmark for these parameters then is that they enable the formulation of laws that simplify the representation of the change that occurs in the configuration of matter - by contrast to merely dressing a list of that change - without losing the information about the change that actually 
happens. The task hence is to specify a dynamical structure such that, for any configuration of matter given as initial condition, the law fixes (or at least puts a constraint on) how the universe would evolve if that configuration were the actual one. The dynamical structure thereby goes beyond the actual configuration of matter: it fixes for any possible configuration of matter what the evolution of the universe would be like if that configuration were actual. It thereby supports counterfactual propositions.

The ontological parsimony of this proposal then shows up in refusing to extend the ontological commitment beyond the primitive ontology - such as the primitive ontology defined by matter points individuated by distance relations and the change of these relations. In other words, the dynamical structure of a physical theory, insofar as it can be construed in terms of its functional role for the primitive ontology, does not call for an additional ontological commitment. Of course, physics explains the motions of bodies by using a geometry and dynamical parameters that figure in laws. However, the argument for an ontological commitment to the geometrical space and the dynamical parameters employed in physical theories cannot simply be that they figure in our best physical theories. Reading the ontology off from the mathematical structure of physical theories would be begging the question of an argument for ontological commitments that go beyond what is minimally sufficient to account for the phenomena in a scientific realist vein, namely the commitment to a primitive ontology as given by the two axioms of distance relations individuating matter points and the change in these relations. In a metaphysics based on science, the argument can only be that by subscribing to ontological commitments that go beyond that minimum, one achieves a gain in explanation.

However, our scientific understanding of the world comes to an end once the salient patterns in the change of the distances among the basic objects are reached, such as e.g. attractive particle motion. In whatever way one spells out in philosophy of science what explanation means - covering law, causal explanation, unification, just to name the most prominent accounts - explanation is achieved when one shows how a phenomenon that calls for an explanation falls into a common pattern (such as e.g. the pattern of attractive motion). To vindicate scientific explanations, no ontological commitment going beyond the minimal commitments spelled out in the previous section is called for (see Loewer 2012). Subscribing to an ontological commitment with respect to the parameters that are introduced in terms of their function for the particle motion does not amount to a gain in explanation. Quite to the contrary, it entails serious drawbacks that come with ontological surplus structure.

The argument for this claim is the one illustrated in Molière's piece Le malade imaginaire: one does not explain why people fall asleep after the consumption of opium by subscribing to an ontological commitment to a dormitive virtue of opium, because that dormitive virtue is defined in terms of its functional role to make people fall asleep after the consumption of opium. By the same token, one does not obtain a gain in explaining attractive particle motion by subscribing to an ontological commitment to gravitational mass as a property of the particles, because mass is defined in terms of its functional role of making objects attract one another as described by the law of gravitation. Of course, mass, charge and the like are fundamental and universal physical parameters, by contrast to the dormitive virtue of opium. But the point is that they are defined in terms of the functional role that they exert for the particle motion. Why do objects move as they do? Because they have properties whose function it is to make them move as they do. An ontological commitment to such properties 
does not yield a gain in explanation. The same holds for forces, fields, wave functions, an ontic structure of entanglement in quantum physics, laws conceived as primitive, etc. It also applies to geometry: it is no gain in explanation to trace the characteristic features of the distance relation back to the geometry of an absolute space, because that geometry is defined such that it allows for the conception of distances in that space.

It is true that by tracing the distance relations back to an absolute space, or the change in the distance relations back to properties of the particles that are dispositions for that very change, the characteristic features of the distance relations as well as those of the patterns of the change in them come out as necessary instead of contingent. However, shifting the status of something from contingent to necessary does not amount to a gain in explanation. Quite to the contrary, one only faces drawbacks that come with the commitment to a surplus structure in the ontology in the guise of an absolute space, fundamental dispositional properties of the particles, ontic dynamical structures of entanglement, etc.: differences with respect to absolute space that do not make a difference in the configuration of matter, questions such as how an object can influence the motion of other objects across space in virtue of properties that are intrinsic to it, how a wave function defined on configuration space can pilot the motion of matter in physical space, etc. (see Esfeld and Deckert 2017, ch. 2.3, for a detailed argument).

The stance that results from these arguments can be dubbed Super-Humeanism: on standard Humean metaphysics (e.g. Lewis 1986a, introduction), the ontology, the Humean mosaic, consists in spatio-temporal relations among points and intrinsic properties instantiated at those points. The Super-Humean deletes the commitment to a substantival space-time and to intrinsic properties, thus avoiding the surplus structure in the ontology that comes with these commitments. Hence, the Super-Humean maintains that there are only sparse points that then are matter points with distance relations individuating these points and change in the distance relations. The laws of physics are our means to represent the salient patterns in that change in a system that strikes the best balance between being simple and being informative about that change. In order to do so, dynamical parameters that enable the formulation of laws have to be introduced in terms of the functional role that they play for the change in the distance relations among the matter points.

However, introducing these parameters does as such not lead to an improvement in simplicity. Values for them have to be fixed as initial conditions along with the relative particle positions. Doing so may turn out to be as complicated and messy as specifying initial positions of the matter points (consider e.g. the case of the wave function in quantum physics). The gain in simplicity is that by figuring out these further parameters over and above the relative particle positions in an initial configuration, one determines an initial condition that can be inserted as input into a law so that one obtains as output (in the deterministic case) a description of the whole past and future evolution of the particle configuration (cf. the distinction between two types of physical state in Bhogal and Perry 2015 - one type that consists only in the relative particle positions, and a further, broader type that includes what has to be specified as initial condition in addition to that). The argument for not subscribing to an additional ontological commitment to these parameters - beyond the commitment to relative particle positions and their change - is that one can introduce these parameters in terms of their functional role for the change in the relative particle positions (so that they do not have to be endorsed as basic) and that in refraining from an additional ontological commitment to them, one avoids a commitment to surplus structure. 
On the one hand, the metaphysics of matter points individuated by distance relations and the change of these relations is a robust scientific realism: it remains stable from ancient Greek atomism to today's physics. It is not hit by the stock arguments against scientific realism from pessimistic meta-induction and underdetermination of theory by evidence, since these arguments concern the change in and the underdetermination of the dynamical structure of physical theories, but not the scientific realist ontology. On the other hand, that ontology, although based on science, may appear pretty much like an a priori, armchair metaphysics: all the experimental evidence that we always had and will ever have are relative positions of discrete objects and their change (including dots on a screen, pointer positions, detector clicks, etc.). Consequently, it will always be possible to adopt the stance that whatever dynamics a future physical theory introduces is a dynamics for the change in the relative positions of basic, discrete objects. In other words, it will always be possible to maintain that whatever new parameters a future physical theory conceives, they come in through the functional role that they play for the change in the relative positions of basic discrete objects.

However, this is not the way in which the ontology defined by the two axioms of matter points individuated by distance relations and change of these relations is conceived as a science based, naturalized metaphysics. The ambition of this metaphysics is to stand out as the best account of the ontological commitments of our physical theories. This has to be established for each physical theory separately, and it may fail in the case of future physical theories. Thus, one has to vindicate Leibnizian relationalism as the overall best proposal for the ontological commitments of classical mechanics (see Huggett 2006), show how this proposal applies to relativistic physics as well (see Vassallo and Esfeld 2016, Vassallo et al. 2017). Furthermore, one has to argue that an ontology of permanent particles being characterized only by their relative positions and the change of these positions makes out the best solution to the measurement problem in quantum physics, both for quantum mechanics (see Miller 2014, Esfeld 2014, Callender 2015, Bhogal and Perry 2017) and for quantum field theory (see Colin and Struyve 2007, Deckert et al. 2017) (see the book Esfeld and Deckert 2017 for setting out this case for both classical and quantum physics).

\section{Beyond physics}

The Canberra plan provides a clear roadmap for both ontology and epistemology. As far as ontology is concerned, the task is to specific a basic set of entities such that everything else can be located in that set and hence be vindicated as being identical with something in that set. Thus, on the naturalized metaphysics sketched out in the two preceding sections, everything in the physical world is identical with a configuration of matter points that is characterized only by the relative distances among the matter points and the change in these distances. The task then is to find out which configurations of matter points are, for instance, water molecules, stones, trees, tigers, tables, etc. In detecting this, the crucial issue is not a configuration at a time, but the salient patterns of relative change in the distance relations in the configuration in question. These patterns provide for the stability of the macroscopic objects with which we are familiar. For instance, an ephemeral table-shaped configuration of matter points would not be a table; only a stable such configuration is a table. As argued in the two preceding sections, to obtain such stable configurations, all that is needed are certain patterns in the particle motion, but not an ontological commitment to intrinsic properties of the basic objects (see also Dickson 2000). 
As regards epistemology, all further notions apart from the primitive ones of matter points, distance relations and the change of these relations come in through a definition in terms of their functional role for the change in the distance relations. Consequently, they are defined in terms of the basic notions. Given these functional definitions, all the true propositions that employ terms that are thus defined - e.g. all the true propositions employing terms such as "mass" and "charge" - then are entailed by the propositions that describe the universal configuration of matter and its change in terms of the primitive notions. The naturalized metaphysics set out in this paper does not maintain that these definitions are an a priori affair of conceptual analysis. They are rather a matter of scientific investigation of salient patterns of motion in the world. Consequently, the entailment is not a priori. But the crucial point is that there is entailment given the description of the world in terms of the basic notions and the functional definitions of all the further notions.

The Canberra plan can of course be applied beyond the domain of the natural sciences. The crucial issue is the functional definition of the relevant concepts in terms of their functional role for, in the last resort, particle motion. Consider mental concepts: there is no question any more today of behaviourism, that is, of defining mental concepts directly in terms of the effects on the bodily motions of persons. Nonetheless, functionalism in the philosophy of mind is the successor of behaviourism (see e.g. Lewis 1966, section III). The functional definition of every single mental concept can include other mental concepts; but in the end, the functional definition of the whole cluster of mental concepts is one in terms of their causal role for the behaviour of the person, that is, for the change in the relative positions of the particles making up the person's body and its environment. This causal role functionalism is to be contrasted with a normative functionalism according to which the functional definition of mental concepts is an affair of indicating their role in a normative network of justifications (giving and asking for reasons) with that normative network constituting a realm of its own (such a normative functionalism can be traced back to Sellars 1956; for a contemporary elaboration, see notably Brandom 1994). Once one has functional definitions in terms of causal roles for in the last resort particle motion at one's disposal, the true propositions employing the concepts defined in that way are entailed by the description of the particle configuration of the universe and its change. Consequently, the referents of those definitions are identical with certain sub-configurations of particles and their change, insofar as that change manifests certain stable regularities - although it may be quite a complicated (if not desperate) affair of empirical research to detect these sub-configurations.

The crucial issue hence is whether all the concepts employed in the description of what there is admit of a definition in terms of their causal role for a basic ontology defined exclusively in terms of primitive physical notions. Functional definitions of this kind are undisputed in the natural sciences: it would be odd, for instance, to postulate a heat stuff to account for thermodynamical phenomena, since these can be defined functionally in terms of changes in molecular motion. By the same token, it would be odd to postulate an élan vital to account for organisms and their reproduction. Since the advent of molecular biology, the evolution of organisms and their reproduction can be accounted for in terms of molecular biology so that functional definitions in terms of causal roles for, in the last resort, particle motion are vindicated. There is no explanatory gap here.

However, when it comes to consciousness as well as rationality and the normativity and freedom that are linked to rationality, one may maintain that there is an explanatory gap in the 
sense that functional definitions in terms of, in the last resort, changes in the configuration of matter do not capture what is characteristic of mental phenomena (see Levine 1983). Once one has understood the science, it is obvious how a functional definition of, for instance, water in terms of the effects on the interaction of $\mathrm{H}_{2} \mathrm{O}$ molecules captures and explains the characteristic phenomenal features of water and how a functional definition of organisms captures and explains their reproduction, including the link from genotypes to phenotypes. However, it is not obvious - at least not obvious in the sense of these paradigmatic examples - what the qualitative character of conscious experience, or the normativity that comes with rationality have to do with molecular motion in the brain.

Going beyond physics, the Canberra plan is not tied to a physicalist ontology. Its rationale rather is the one of setting a clear standard for ontology and thereby making evident the consequences that follow from endorsing a physicalist ontology as well as the consequences that one has to accept in any ontology that goes beyond physicalist commitments. The point is that any such further commitments come in as new primitives. For instance, in case the mental cannot be functionally defined on the basis of a primitive ontology of matter in motion, then an ontological commitment to the mental is called for over and above the ontological commitment to a primitive physical ontology. Such an ontological commitment then is as fundamental as the commitment to a primitive physical ontology, although the mental may only exist in certain systems in the universe and only at a certain period of time (cf. the Canberra plan of Chalmers 2012 in which conscious experience figures as a further primitive beyond the physical ones). In general, whatever does not come in as being located in the basic set of entities - i.e. in a physicalist ontology as being identical with a specific subconfiguration of particles and their characteristic motion - and hence not as being entailed by the description of the particle configuration of the universe and its change in terms of the basic, primitive notions is itself a further primitive ingredient of the ontology.

The alternative to this rigour of the Canberra plan is the attempt to introduce notions that are weaker than identity with the intention of thus eschewing the commitment to further ontological primitives beyond the physicalist ones. Emergence is the most popular such notion. There is a trivial sense of emergence in that new things come up in the evolution of the universe. However, if what emerges can be functionally defined on the basis of the ontology that is endorsed as primitive, then there is no emergence in any philosophically relevant sense, namely in the sense of something that ontologically goes beyond what was recognized as primitive. If what emerges cannot be thus defined, then we have a situation in which something that is included in the ontology originally admitted as primitive is correlated with something of which it is maintained that it cannot be defined in terms of its causal role for changes in something that is included in that ontology. Consider as a pertinent example a sub-configuration of particles that is a brain and conscious experience conceptualized in terms of qualia. However, we then have a primitive correlation. Saying that conscious experience emerges from neuronal particle configurations does not go beyond stating that there is a (systematic) correlation between certain particle configurations and certain phenomena that do not consist in excerizing a certain causal role for changes in the particle configurations in question. Thus, when it comes to ontology, one is committed to more than what was originally admitted as primitive (i.e. matter in motion). Consequently, there then are further primitives that hence have the same ontological status as the original primitives, and the notion of emergence is of no use when it comes to ontology. 
The same goes for the notion of grounding. According to its proponents (e.g. Schaffer 2009 , Fine 2012), grounding is not identity. Nevertheless, if $A$ grounds $B$, then $B$ is supposed to come in for free - that is to say, recognizing the existence of $B$ requires no ontological commitment that goes beyond the commitment to $A$. However, it is not evident to say the least how this can be so. Again, what we have is a (systematic) correlation between two types of phenomena, such as, for instance, particle configurations that are brains and conscious experiences conceptualized in terms of qualia. It is not clear what work the notion of grounding does to explain this correlation by turning it into a priority of $A$ (the brain) over $B$ (the qualia) - unless in the trivial sense that there are particle configurations everywhere and all the time and that only some particle configurations in the universe, which moreover occur only at some times in the evolution of the universe, are correlated with qualia. Nonetheless, the correlation then remains primitive. Hence, again, if $A$ is not identical with $B$, then there is something that makes $B$ different from $A$, something which is not included in $A$, so that an ontological commitment going beyond $A$ is necessary, and that commitment has to be added to the commitments that were originally endorsed as primitive.

In sum, the merit of the Canberra plan is to set clear standards for metaphysics. These standards can be illustrated - and the Canberra plan be carried out in concrete terms - for the domain of the natural sciences. Going beyond that domain, the Canberra plan points out what commitments have to be undertaken - namely either to make do with the ontology admitted as primitive for the physical domain or to enlarge that ontology by further primitives that then have the same status as the original primitives - and what the consequences are in each of these two scenarios.

\section{References}

Belot, Gordon (2001): “The principle of sufficient reason”. Journal of Philosophy 98, pp. 55-74.

Bhogal, Harjit and Perry, Zee R. (2017): "What the Humean should say about entanglement”. Noûs 51, pp. 7494.

Brandom, Robert B. (1994): Making it explicit. Reasoning, representing, and discursive commitment. Cambridge (Massachusetts): Harvard University Press.

Callender, Craig (2015): “One world, one beable”. Synthese 192, pp. 3153-3177.

Chalmers, David (2012): Constructing the world. Oxford: Oxford University Press.

Colin, Samuel and Struyve, Ward (2007): “A Dirac sea pilot-wave model for quantum field theory”. Journal of Physics A 40, pp. 7309-7341.

Deckert, Dirk-André, Esfeld, Michael and Oldofredi, Andrea (2017): “A persistent particle ontology for QFT in terms of the Dirac sea". Forthcoming in British Journal for the Philosophy of Science, preprint arXiv:1608.06141[physics.hist-ph]

Dickson, Michael (2000): “Are there material objects in Bohm's theory?” Philosophy of Science 67, pp. 704710.

Esfeld, Michael (2014): “Quantum Humeanism, or: physicalism without properties”. Philosophical Quarterly 64, pp. 453-470.

Esfeld, Michael and Deckert, Dirk-André (2017): A minimalist ontology of the natural world. New York: Routledge.

Feynman, Richard P., Leighton, Robert B. and Sands, Matthew (1963): The Feynman lectures on physics. Volume 1. Reading (Massachusetts): Addison-Wesley.

Fine, Kit (2012): “A guide to ground”. In F. Correia and B. Schnieder (eds.): Metaphysical grounding. Cambridge: Cambridge University Press. Pp. 37-80.

French, Steven (2014): The structure of the world. Metaphysics and representation. Oxford: Oxford University Press. 
Hacking, Ian (1975): "The identity of indiscernibles". Journal of Philosophy 72, pp. 249-256.

Hall, Ned (2009): "Humean reductionism about laws of nature". Unpublished manuscript, http://philpapers.org/rec/HALHRA

Heil, John (2012): The universe as we find it. Oxford: Oxford University Press.

Huggett, Nick (2006): “The regularity account of relational spacetime”. Mind 115, pp. 41-73.

Jackson, Frank (1994): “Armchair metaphysics”. In: J. O’Leary-Hawthorne and M. Michael (eds.): Philosophy in mind. Dordrecht: Kluwer. Pp. 23-42.

Jackson, Frank (1998): From metaphysics to ethics. A defence of conceptual analysis. Oxford: Oxford University Press.

Ladyman, James and Ross, Don (2007): Every thing must go. Metaphysics naturalized. Oxford: Oxford University Press.

Leibniz, Gottfried Wilhelm (1890): Die philosophischen Schriften von G. W. Leibniz. Band 7. Edited by C. I. Gerhardt. Berlin: Weidmannsche Verlagsbuchhandlung.

Leibniz, Gottfried Wilhelm (2000): G. W. Leibniz and S. Clarke: Correspondence. Edited by Roger Ariew. Indianapolis: Hackett.

Levine, Joseph (1983): "Materialism and qualia: the explanatory gap”. Pacific Philosophical Quarterly 64, pp. 354-361.

Lewis, David (1966): “An argument for the identity theory”. Journal of Philosophy 63, pp. 17-25.

Lewis, David (1970): "How to define theoretical terms". Journal of Philosophy 67, pp. 427-446.

Lewis, David (1972): "Psychophysical and theoretical identifications". Australasian Journal of Philosophy 50, pp. 249-258.

Lewis, David (1986a): Philosophical papers. Volume 2. Oxford: Oxford University Press.

Lewis, David (1986b): On the plurality of worlds. Oxford: Blackwell.

Lewis, David (2009): "Ramseyan humility". In: D. Braddon-Mitchell and R. Nola (eds.): Conceptual analysis and philosophical naturalism. Cambridge (Massachusetts): MIT Press. Pp. 203-222.

Loewer, Barry (2012): "Two accounts of laws and time”. Philosophical Studies 160, pp. 115-137.

Lowe, E. Jonathan (2016): “The are (probably) no relations". In: A. Marmodoro and D. Yates (eds): The metaphysics of relations. Oxford: Oxford University Press. Pp. 100-112.

Mach, Ernst (1919): The science of mechanics: a critical and historical account of its development. Fourth edition. Translation by Thomas J. McCormack. Chicago: Open Court.

Miller, Elizabeth (2014): "Quantum entanglement, Bohmian mechanics, and Humean supervenience". Australasian Journal of Philosophy 92, pp. 567-583.

Quine, Willard Van Orman (1948): “On what there is”. Review of Metaphysics 2, pp. 21-38.

Schaffer, Jonathan (2009): "On what grounds what". In D. Manley, D. Chalmers and R. Wasserman (eds.): Metametaphysics: new essays on the foundations of ontology. Oxford: Oxford University Press. Pp. 347383.

Sellars, Wilfrid (1956): "Empiricism and the philosophy of mind". In: H. Feigl and M. Scriven (eds.): The foundations of science and the concepts of psychology and psychoanalysis. Minneapolis: University of Minnesota Press. Pp. 253-329.

Vassallo, Antonio and Esfeld, Michael (2016): "Leibnizian relationalism for general relativistic physics". Studies in History and Philosophy of Modern Physics 55, pp. 101-107.

Vassallo, Antonio, Deckert, Dirk-André and Esfeld, Michael: "Relationalism about mechanics based on a minimalist ontology of matter". European Journal for Philosophy of Science 7, pp. 299-318. 